\title{
Isolating bronchial epithelial cell preparations from gross lung specimens
}

\author{
Yong-Jae Kwon • Seog Joo Lee • Jae Soo Koh • \\ Young-Joon Kim • Jong Ho Park
}

Received: 12 February 2009 / Accepted: 2 April 2009 / Editor: J. Denry Sato

(C) The Author(s) 2009. This article is published with open access at Springerlink.com

\begin{abstract}
Tissue microdissection is appropriate for separating pure cells from heterogeneous tissues. Recently, we have focused on whole-genome DNA methylation patterns of lung squamous cell carcinoma (SCC) and needed to obtain the appropriate counterpart cells of lung SCC. However, in some regions of human tissues, such as in bronchial epithelium, it is difficult to apply tissue microdissection as a means to isolate pure cells from a heterogenous mixture of cells. Accordingly, we developed the pop brush method to retrieve sufficient amounts of pure bronchial epithelium from gross lung specimens and this method enables us to study epigenetic variations of lung SCC.
\end{abstract}

\footnotetext{
J. H. Park $(\bowtie)$

Department of Thoracic Surgery, Korea Cancer Center Hospital, Korea Institute of Radiological and Medical Sciences,

215-4 Gongneung-Dong, Nowon-Gu,

Seoul 139-706, Republic of Korea

e-mail: jhpark@kcch.re.kr

Y.-J. Kwon· S. J. Lee

Department of Clinical Research, Korea Cancer Center Hospital, Korea Institute of Radiological and Medical Sciences,

Seoul, Republic of Korea
}

\section{J. S. Koh}

Department of Pathology, Korea Cancer Center Hospital, Korea Institute of Radiological and Medical Sciences, Seoul, Republic of Korea

\section{Y.-J. Kim $(\bowtie)$}

Department of Biochemistry, Yonsei University,

Seoul, Republic of Korea

e-mail: yjkim@yonsei.ac.kr
Keywords Tissue microdissection - Whole-genome methylation patterns analysis · Lung squamous cell carcinoma (SCC) $\cdot$ Pop brush method $\cdot$ Bronchial epithelial cell as a counterpart of lung SCC

Tissue microdissection is a useful tool for separating pure cells from heterogeneous tissues. Since the development of this technique, the isolation of a homogeneous population of pure cells from a heterogeneous tissue section has allowed for more accurate results following whole-genomescale analyses such as gene expression profiling and wholegenome methylation analyses (Bleul et al. 2001; Kitahara et al. 2001; Grove et al. 2002; Miura et al. 2002; Nie et al. 2002; Sato et al. 2003). Recently, we have focused on the whole-genome methylation pattern analysis of lung squamous cell carcinoma (SCC). The most established developmental stages of lung SCC are as follows: (1) in response to outside toxins, normal bronchial epithelium may undergo hyperplasia and squamous metaplasia; (2) the formation of dysplasia, leading to squamous cell carcinoma in situ; and finally (3) the development of invasive squamous cell carcinoma. In order to isolate the appropriate counterpart cells from cancer cells, we tried to separate squamous cancer cells of lungs and normal epithelial from the surgical specimens of lung SCC patients from whom informed consent was obtained. In this manner, we successfully obtained pure cancer cells by tissue microdissection. However, since normal epithelial cells reside in just the most outer membrane within a very narrow region of bronchial walls shown as Fig. 1a, it was difficult to obtain sufficient amounts of these cells for the whole-genome methylation assay. Therefore, it has been considered labor- 

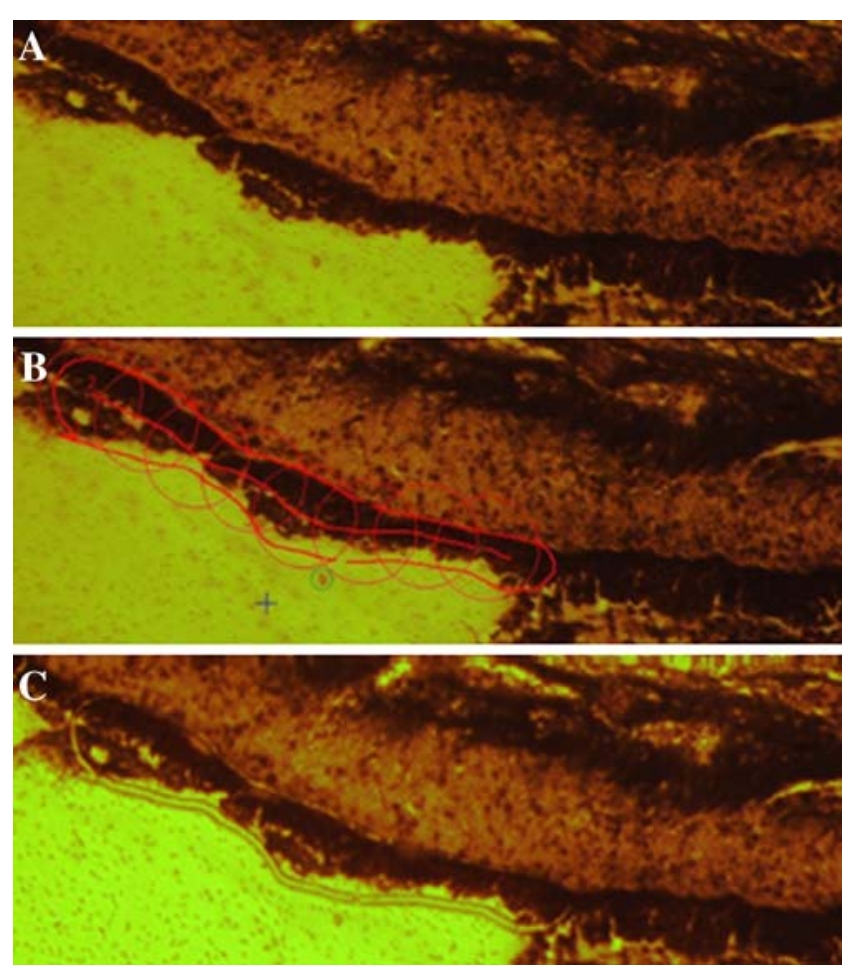

Figure 1. The limitations in obtaining homogenous bronchial epithelial cells from gross lung specimens by tissue microdissection. Since epithelial cells line the most outer membrane of lung tissues and reside within a very narrow region, it was hard to isolate them using a UV laser and IR spotting. In (b), the red line is the UV cutting line and the red circles represent IR spotting. As shown in (c), none of the epithelial cells were captured using this technique. (a) Gross lung specimen before tissue microdissection. (b) A view of tissue microdissection showing the UV cutting line and IR spots. (c) Gross lung specimen after tissue microdissection.

intensive and time-wasteful to collect bronchial epithelial cells by tissue microdissection. Additionally, as shown in Fig. $1 b, c$, even though microdissection was completed, bronchial epithelium remained because infrared spotting was not strong enough to detach the cells from the slide in these narrow regions. Here, we developed the pop brush method to separate only the normal bronchial epithelial cells from lung tissues that were not contaminated with other type of cells.

1. Bronchial washing to clean the inner membrane of lungs

Immediately following dissection, lung tissues were washed first with distilled water and then with phosphatebuffered saline (PBS) containing antibiotics. The inner parts of all bronchi were washed using a syringe and all traces and sputum inside the bronchi were removed.

2. Scrape the inner membrane of the bronchi to obtain epithelial cells

We developed the use of a pop brush to scrape the inner membrane of bronchi and to obtain epithelial cells. At first, lung tissues were immobilized with a syringe on the plate. Next, we scraped the inner wall of the bronchus with a pop brush keeping tension of lung tissues. We usually repeated bronchial scraping to isolate enough epithelial cells for analysis.

\section{Preparing genomic DNA from pop brush}

After scraping was completed, the end of the pop brush was cut and put into ATL buffer for preparing genomic DNA as shown in Fig. $2 a$. Next, we followed the "Isolation of genomic DNA from swabs" protocol in the QIAamp DNA Micro Handbook (Qiagen, Hilden, Germany). Ultimately, more than $10-\mu \mathrm{g}$ genomic DNA was obtained from normal epithelial cells and this amount was sufficient for analyzing genomic DNA by polymerase chain reaction (PCR).

In order to validate the complete and delicate removal of epithelial cells from bronchi, we made frozen slides of the bronchial tissue used for scraping. Next, staining with hematoxylin and eosin dye was performed and the slides were observed using a light microscope. In this manner, the removal of epithelial cells from the inner membrane of the bronchi was confirmed as shown in Fig. $2 b$.

The QIAamp DNA micro kit was used to isolate the genomic DNA from normal epithelial cells and PCR was performed on these specimens using a primer pair specific to human C-Jun.

For these reactions, 34 cycles of PCR were performed on $50 \mathrm{ng}$ of genomic DNA. As shown in Fig. $2 c$, a single band was detected following amplification by PCR and separation by gel electrophoresis. These results show that the genomic DNA of normal epithelial cells from bronchi obtained using the technique described is sufficient for further analysis by PCR.

The genomic and epigenomic study of a pure population of cells has been increasingly used to accurately examine various molecular events happening within these cells (Kuzmin et al. 2002; Kwong et al. 2002; Kim et al. 2003; Chan et al. 2004). Often, tissue microdissection is sufficient to obtain a pure population of cells from a heterogeneous mixture of cells. Some regions, however, such as those containing bronchial epithelial cells are elongated 

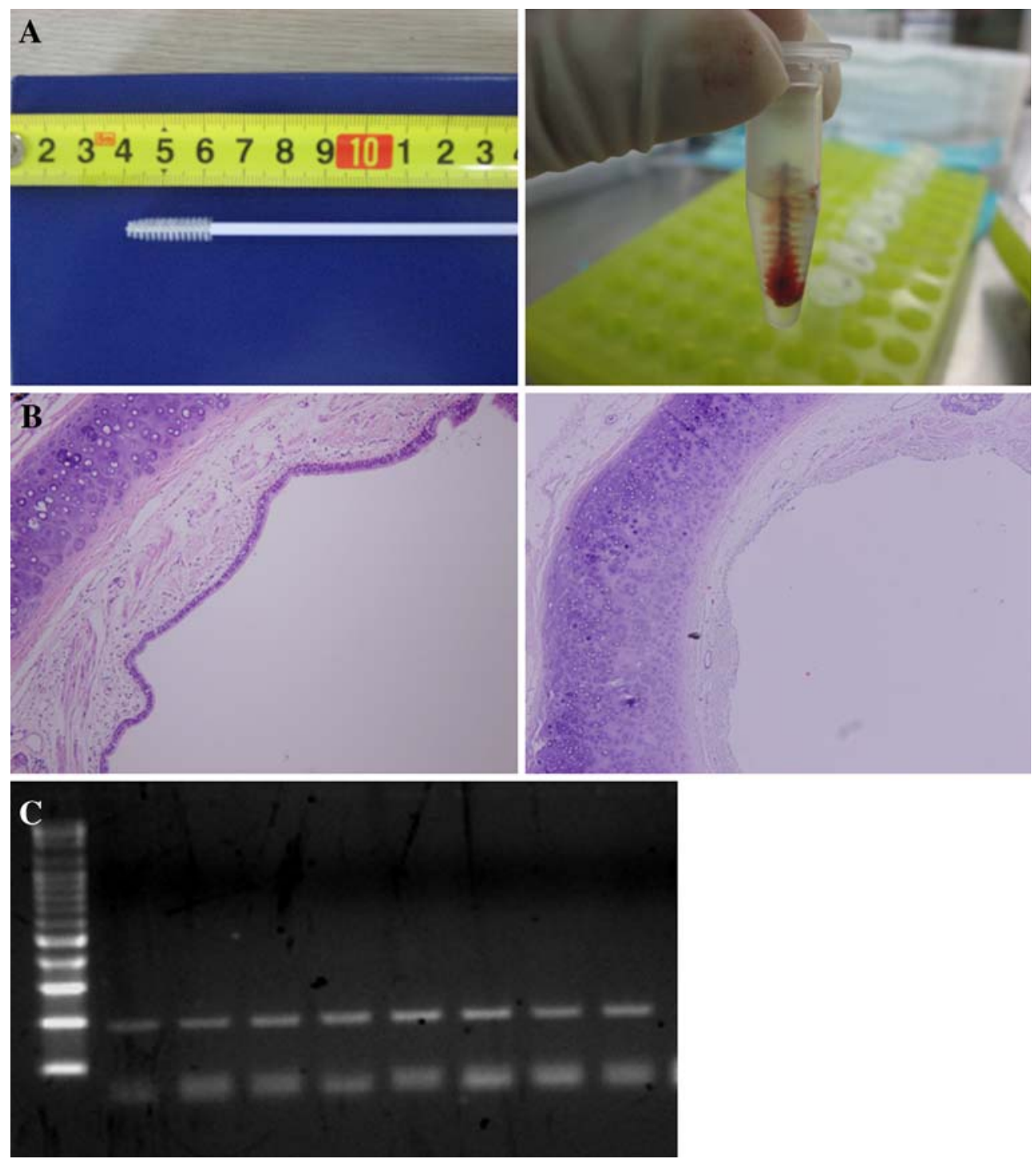

Figure 2. Validating the preparation of normal bronchial epithelial cells from gross lung specimens. We confirmed the genomic DNA preparation by comparing microscopic observations of the lung bronchus epithelium before and after scraping and by using PCR. (a) Left panel, pop brush; right panel; scraped brush in the tube containing buffer. (b) Left panel: before scraping; right panel: after scraping. (c) PCR results with c-JUN primer set using epithelial cell genomic DNA as a template. c-JUN primer sequences: forward, 5-TTGTTTGTTTGGGTATCCTG-3, and reverse, 5-ATGCAGAAAAGAGGTTAGGG-3. and too narrow to allow for tissue microdissection, particularly since cellular component such as genomic DNA and RNA may be damaged by the UV light used during this technique. Also, this technique is time consuming and requires many tissue slides to obtain sufficient amounts of bronchial epithelium required for wholegenome analyses. Here, we developed the pop brush method to obtain sufficient amounts of bronchial epithelial cells from gross lung tissues. Finally, we suggest that this method provides us with enough homogeneous normal counterparts of lung SCC for studying genetic and epigenetic variations.

Acknowledgment The Korea Institute of Radiological and Medical Sciences Institutional Review Board as the institutional review board approved the human protocol of these experiments. 
Open Access This article is distributed under the terms of the Creative Commons Attribution Noncommercial License which permits any noncommercial use, distribution, and reproduction in any medium, provided the original author(s) and source are credited.

\section{References}

Bleul C. C.; Boehm T. Laser capture microdissection-based expression profiling identifies PD1-ligand as a target of the nude locus gene product. Eur. J. Immunol 31: 2497-2503; 2001. doi:10.1002/1521-4141(200108)31:8<2497::AIDIMMU2497>3.0.CO;2-J.

Chan Y.; Fish J. E.; D’Abreo C.; Lin S.; Robb G. B.; Teichert A. -M.; Karantzoulis-Fegaras F.; Keightley A.; Steer B. M.; Marsden P. A. The cell-specific expression of endothelial nitric-oxide synthase- - a role for DNA methylation. J. Biol. Chem 279: 35087-35100; 2004. doi:10.1074/jbc.M405063200.

Grove A. D.; Prabhu V. V.; Young B. L.; Lee F. C.; Kulpa V.; Munson P. J.; Kohn E. C. Both protein activation and gene expression are involved in early vascular tube formation in vitro. Clin. Cancer Res 8: 3019-3026; 2002.

Kim J.; Herlyn D.; Wong K.; Park D. -C.; Schorge J. O.; Lu K. H.; Skates S. J.; Cramer D. W.; Berkowitz R. S.; Mok S. Identification of epithelial cell adhesion molecule autoantibody in patients with ovarian cancer. Clin. Cancer Res 9: 4782-4791; 2003.
Kitahara O.; Furukawa Y.; Tanaka T.; Kihara C.; Ono K.; Yanagawa R.; Nita M. E.; Takagi T.; Nakamura Y.; Tsunoda T. Alterations of gene expression during colorectal carcinogenesis revealed by cDNA microarrays after laser-capture microdissection of tumor tissues and normal epithelia. Cancer Res 61: 3544-3549; 2001.

Kuzmin I.; Gillespie J. W.; Protopopov A.; Geil L.; Dreijerink K.; Yang Y.; Vocke C. D.; Duh F. -M.; Zabarovsky E.; Minna J. D.; Rhim J. S.; Emmert-Buck M. R.; Linehan W. M.; Lerman M. I. The RASSF1A tumor suppressor gene is inactivated in prostate tumors and suppresses growth of prostate carcinoma cells. Cancer Res 62: 3498-3502; 2002.

Kwong J.; Lo K. -W.; To K. -F.; Teo P. M. L.; Johnson P. J.; Huang D. P. Promoter hypermethylation of multiple genes in nasopharyngeal carcinoma. Clin. Cancer Res 8: 131-137; 2002.

Miura K.; Bowman E. D.; Simon R.; Peng A. C.; Robles A. I.; Jones R. T.; Katagiri T.; He P.; Mizukami H.; Charboneau L.; Kikuchi T.; Liotta L. A.; Nakamura Y.; Harris C. C. Laser capture microdissection and microarray expression analysis of lung adenocarcinoma reveals tobacco smoking- and prognosis-related molecular profiles. Cancer Res 62: 3244-3250; 2002.

Nie Y.; Liao J.; Zhao X.; Song Y.; Yang G.; Wang L. -D.; Yang C. S. Detection of multiple gene hypermethylation in the development of esophageal squamous cell carcinoma. Carcinogenesis 23: 1713-1720; 2002. doi:10.1093/carcin/23.10.1713.

Sato N.; Maitra A.; Fukushima N.; van Heek N. T.; Matsubayashi H.; Iacobuzio-Donahue C. A.; Rosty C.; Goggins M. Frequent hypomethylation of multiple genes overexpressed in pancreatic ductal adenocarcinoma. Cancer Res 63: 4158-4166; 2003. 